\title{
Obligate sugar oxidation in Mesotoga spp., phylum Thermotogae, in the presence of either elemental sulfur or hydrogenotrophic sulfate-reducers as electron acceptor
}

\author{
Khaled Fadhlaoui, ${ }^{1,2}$ Wagdi Ben Hania, ${ }^{1}$ \\ Fabrice Armougom, ${ }^{1}$ Manon Bartoli, ${ }^{1}$ \\ Marie-Laure Fardeau, ${ }^{1}$ Gaël Erauso, ${ }^{1}$ \\ Gaël Brasseur, ${ }^{3}$ Corinne Aubert, ${ }^{3}$ Moktar Hamdi, ${ }^{2}$ \\ Céline Brochier-Armanet, ${ }^{4}$ Alain Dolla ${ }^{3 *}$ and \\ Bernard Ollivier $^{1 \text { ** }}$ \\ ${ }^{1}$ Aix Marseille Univ, Université de Toulon, CNRS, IRD, \\ MIO, Marseille, France. \\ 'Laboratoire d'Ecologie et de Technologie Microbienne, \\ Institut National des Sciences Appliquées et de \\ Technologie, Université de Carthage, Centre Urbain \\ Nord, Tunis, BP 676, 1080, Tunisia. \\ ${ }^{3}$ Aix Marseille Univ, CNRS, LCB, Marseille, France. \\ ${ }^{4}$ Université de Lyon; Université Lyon 1; CNRS; \\ UMR5558, Laboratoire de Biométrie et Biologie \\ Evolutive, 43 boulevard du 11 novembre 1918, \\ Villeurbanne, F-69622, France.
}

\section{Summary}

Mesotoga prima strain PhosAc3 is a mesophilic representative of the phylum Thermotogae comprising only fermentative bacteria so far. We show that while unable to ferment glucose, this bacterium is able to couple its oxidation to reduction of elemental sulfur. We demonstrate furthermore that $M$. prima strain PhosAc3 as well as M. prima strain MesG1 and Mesotoga infera are able to grow in syntrophic association with sulfatereducing bacteria (SRB) acting as hydrogen scavengers through interspecies hydrogen transfer. Hydrogen production was higher in M. prima strain PhosAc3 cells co-cultured with SRB than in cells cultured alone in the presence of elemental sulfur. We propose that the efficient sugar-oxidizing metabolism by $M$. prima strain PhosAc3 in syntrophic association with a hydrogenotrophic sulfate-reducing bacterium

Received 5 May, 2017; revised 2 November, 2017; accepted 6

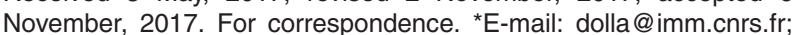

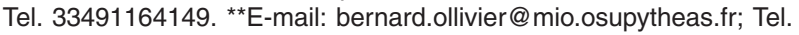
33491828586 can be extrapolated to all members of the Mesotoga genus. Genome comparison of Thermotogae members suggests that the metabolic difference between Mesotoga and Thermotoga species (sugar oxidation versus fermentation) is mainly due to the absence of the bifurcating [FeFe]-hydrogenase in the former. Such an obligate oxidative process for using sugars, unusual within prokaryotes, is the first reported within the Thermotogae. It is hypothesized to be of primary ecological importance for growth of Mesotoga spp. in the environments that they inhabit.

Introduction

For a long time, cultivated members of Thermotogae, a deep-branching phylum within Bacteria (Huber and Hannig, 2006; Cappelletti et al., 2014), were known to be essentially thermophilic to hyperthermophilic. This situation changed when 16S rRNA gene sequences were reported in many mesothermic environments, suggesting that mesophilic (Mesotoga) Thermotogae exist (Nesbø et al., 2006). The first mesophilic representative of Thermotogae (Mesotoga strain PhosAc3) was cultivated in 2011 (Ben Hania et al., 2011). Thereafter, two Mesotoga species, M. prima strain MesG1.Ag.4.2 ${ }^{\top}$ (Nesbø et al., 2012) and M. infera strain VNs100 ${ }^{\top}$ (Ben Hania et al., 2013) were characterized while Mesotoga strain PhosAc3 was recently recognized as a $M$. prima strain (Ben Hania et al., 2015). Phylogenetic analyses of $16 \mathrm{~S}$ rRNA genes revealed that several distinct uncultured lineages of mesophilic Thermotogae may exist and thus that adaptation to mesothermic environments occurred several times independently during the diversification of Thermotogae (Nesbø et al., 2010; Ben Hania et al., 2011). Therefore, Mesotoga spp. are of noticeable interest to understand bacterial evolution from thermophily to mesophily. To date, beside the genus Mesotoga, Thermotogae comprise 12 other genera including Athalassotoga, Defluviitoga, Fervidobacterium, Geotoga, Kosmotoga, Marinitoga, Mesoaciditoga, Oceanotoga, Petrotoga, Pseudothermotoga, Thermosipho and Thermotoga (Di Pippo et al., 2009; 
Jayasinghearachchi and Lal, 2011; Ben Hania et al., 2012; Reysenbach et al., 2013; Bhandari and Gupta, 2014; Cappelletti et al., 2014; Itoh et al., 2016). They all share an outer sheath-like structure called a 'toga' ballooning over the ends of the cell (e.g. Thermotoga and Thermosipho spp.) (Huber et al., 1986; Antoine et al., 1997). Members of the phylum Thermotogae including the orders Thermotogales, Kosmotogales, Petrotogales and Mesoaciditogales (Bhandari and Gupta, 2014; Itoh et al., 2016) are usually considered as heterotrophic fermentative microorganisms able to use sugars, polysaccharides or complex organic substrates such as peptone and yeast extract. However, in contrast to all Thermotogae and to $M$. prima strain MesG1.Ag.4.2 ${ }^{\top}$ in particular, and despite a very close phylogenetic relatedness with the latter, $M$. prima strain PhosAc3 and $M$. infera strain $\mathrm{VNs} 100^{\top}$ are able to use sugars only in the presence of elemental sulfur as terminal electron acceptor leading to the production of acetate, $\mathrm{CO}_{2}$ and sulfide (around 2 moles of acetate and 4 moles of sulfide produced per mole of glucose consumed) with no or only traces of hydrogen production (less than 1 $\mu \mathrm{M}$ ) (Ben Hania et al., 2013; 2015; Cappelletti et al., 2014). This sulfur-dependent metabolism discovered in these two closely related Mesotoga species does not concern exclusively sugars but also applies to other substrates tested (e.g. lactate and pyruvate) (Ben Hania et al., 2011; 2015) clearly contrasting with typical metabolic features of Thermotogae well known as efficient $\mathrm{H}_{2}$ producing bacteria (over $1 \mathrm{mM}$ ) (Cappelletti et al., 2014). However, determining if sulfur reduction is an ATPyielding reaction in Mesotoga spp. has not been established so far.
Here we show that co-culturing M. prima strain PhosAc3 with hydrogenotrophic sulfate-reducing partners (Desulfovibrio and Desulfotomaculum spp.) in the presence of sulfate as terminal electron acceptors significantly improved glucose oxidation by the former. Such metabolic process was extended to other Mesotoga spp. isolated so far including both $M$. prima strain MesG1.Ag.4. $2^{\top}$ and M. infera strain $\mathrm{VNs}_{100}{ }^{\top}$. We demonstrate two major points regarding Mesotoga species: (i) their dependence on the presence of elemental sulfur as terminal electron acceptor to use sugar, thus indicating that sugars are oxidized rather than fermented and (ii) the efficient syntrophic association with hydrogenotrophic partners able to replace more efficiently elemental sulfur as biological electron acceptor for sugar oxidation. Our data point out an unusual prokaryotic metabolism within the Thermotogae, the syntrophic association with a hydrogenotrophic partner in the absence of elemental sulfur to degrade easily fermentable substrates such as carbohydrates and highlight its possible ecological significance in nature.

\section{Results}

Growth of Mesotoga spp. in pure culture and in co-culture with either sulfate-reducing bacteria or methanogens

When $M$. prima strain PhosAc3 was cultured with glucose as energy source, only a slight glucose consumption was observed even after 250 days of incubation at $37^{\circ} \mathrm{C}$ only (Fig. 1A, Table 1). When elemental sulfur was added in the medium, a slow linear degradation of glucose was measured with $6.57 \pm 0.19 \mathrm{mM}$ of the glucose consumed
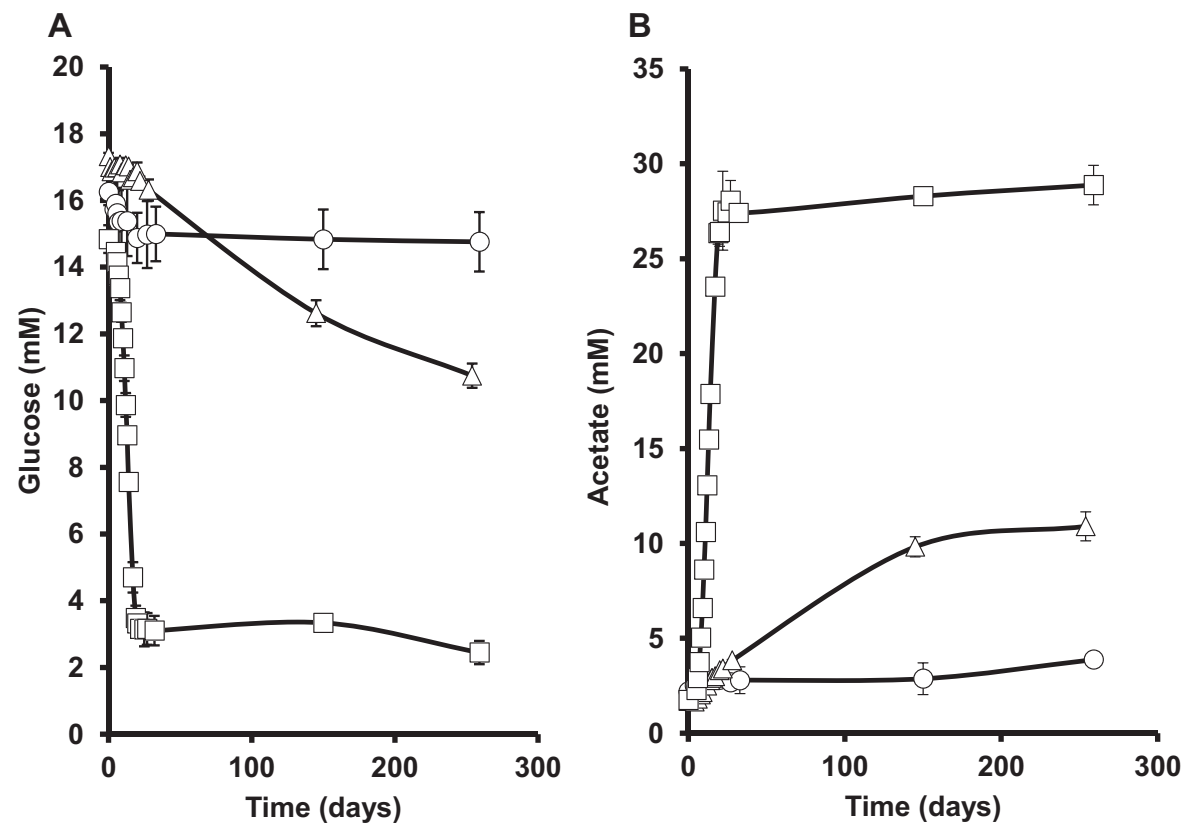

Fig. 1. A. Glucose consumption in pure culture of $M$. prima strain PhosAc3 in either the absence (circle) or presence (triangle) of elemental sulfur $\left(\mathrm{S}^{\circ}\right)$ or in co-culture with D. vulgaris (square) over the time. B. Acetate production in either pure culture of $M$. prima in the absence (circle) or presence (triangle) of elemental sulfur $\left(\mathrm{S}^{\circ}\right)$ or in co-culture with $D$. vulgaris (square) over the time. 
Table 1. End-products quantification of the single and co-cultures of M. prima PhosAc3 and M. prima MesG1Ag4.2T.

\begin{tabular}{|c|c|c|c|c|c|c|}
\hline & $\begin{array}{l}\text { M. prima } \\
\text { PhosAc3 }\end{array}$ & $\begin{array}{l}\text { M. prima } \\
\text { PhosAc3 }+\mathrm{S}^{\circ}\end{array}$ & $\begin{array}{l}\text { M. prima } \\
\text { PhosAc3 } \\
+ \text { D. vulgaris }\end{array}$ & $\begin{array}{l}\text { M. prima } \\
\text { MesG1Ag4.2 }\end{array}$ & $\begin{array}{l}\text { M. prima } \\
\text { MesG1Ag4.2 }{ }^{\top}+\mathrm{S}^{\circ}\end{array}$ & $\begin{array}{l}\text { M. prima } \\
\text { MesG1Ag4.2 } 2^{\top} \\
+ \text { D. salexigens }\end{array}$ \\
\hline Sugar consumed (mM) & $1.50 \pm 0.92$ & $6.57 \pm 0.19$ & $12.39 \pm 0.33$ & $1.00 \pm 0.23$ & $3.27 \pm 0.85$ & $6.65 \pm 1.88$ \\
\hline Acetate produced (mM) & $1.67 \pm 0.21$ & $9.21 \pm 0.13$ & $27.13 \pm 0.45$ & $0.70 \pm 0.41$ & $8.48 \pm 1.96$ & $13.64 \pm 1.75$ \\
\hline $\mathrm{H}_{2} \mathrm{~S}$ produced (mM) & $1.05 \pm 0.25$ & $24.40 \pm 0.30$ & $9.70 \pm 0.85$ & $1.18 \pm 0.41$ & $18.03 \pm 5.16$ & $14.00 \pm 4.52$ \\
\hline
\end{tabular}

(around 33\%) after 250 days of incubation (Fig. 1A, Table 1). Accordingly, only a slight acetate production was measured in the absence of elemental sulfur while $9.21 \pm$ $0.13 \mathrm{mM}$ acetate was produced in its presence after 250 days. The only detected end-products of glucose metabolism were acetate (Fig. 1B), $\mathrm{CO}_{2}$ and sulfide (Table 1). No other volatile fatty acid (e.g. formate, butyrate, etc.) nor organic compound (e.g. lactate, ethanol) was detected in any growth conditions. Surprisingly, only trace amount of hydrogen (around $1 \mu \mathrm{M}$ ) were detected in the gas phase during glucose consumption whatever the presence or the absence of elemental sulfur.

These data clearly showed that $M$. prima strain PhosAc3 was unable to ferment glucose while, in the presence of an external electron acceptor (elemental sulfur), it was able to oxidize it although with a low efficiency. The slight glucose consumption in the absence of elemental sulfur was probably due to the presence of an electron acceptor available in yeast extract since hydrogen was detected only in minor quantities (around $1 \mu \mathrm{M}$ ) in the gas phase.

Because Mesotoga species are often detected in environments where sulfate-reducing bacteria are present (Nesbø et al., 2010), the capability of $M$. prima strain PhosAc3 to grow in syntrophic association with a sulfatereducing bacterium (SRB), Desulfovibrio vulgaris subsp. vulgaris, was tested. As with many other SRB, D. vulgaris gains energy for biosynthesis and growth by coupling oxidation of organic compounds or molecular hydrogen to reduction of sulfate to sulfide (Muyzer and Stams, 2008).

When either $M$. prima strain PhosAc3 or $D$. vulgaris subsp. vulgaris alone was cultured in a glucose/sulfate medium, no growth was observed (Fig. 2A), confirming that this Desulfovibrio species is unable to metabolize sugar. However, when the two bacteria were co-cultured, growth occurred as evidenced by a substantial increase of the optical density (OD) at $580 \mathrm{~nm}$ (Fig. 2A). After only 20 days of incubation, $80 \%$ of the initial glucose was consumed $(\sim 12 \mathrm{mM})$ with the concomitant acetate production of $\sim 25 \mathrm{mM}$ (Fig. 1A and B). The rate of glucose consumption was $0.8 \mathrm{mM}$ glucose consumed/day. After 250 days of incubation, glucose consumption and acetate production only slightly progressed to give $\sim 13 \mathrm{mM}$ glucose consumed and $\sim 28 \mathrm{mM}$ acetate produced (Fig. 1, Table 1). At this time, around $10 \mathrm{mM}$ sulfide had been produced (Table 1). Electron recovery values were $77 \%$ and $85 \%$ when M. prima strain PhosAc3 was grown in the presence of elemental sulfur and $D$. vulgaris as electron acceptors respectively. These values are in agreement with those expected for an anaerobic sugar-oxidizing metabolism leading to acetate as the only produced fatty acid, where about $20 \%$ of the sugar-derived reducing equivalents have been shown to be incorporated into the cells (Thauer et al., 1977; Cord-Ruwish et al., 1986). In contrast, when M. prima strain PhosAc3 was grown in the absence of elemental sulfur, electron recovery was lower (60\%). In this case however, minor amounts of glucose used by M. prima strain PhosAc3 made this value less accurate (Table 1). When another SRB, Desulfotomaculum gibsoniae was used instead of $D$. vulgaris in the co-culture, a similar growth was obtained (data not shown). M. infera strain VNs $100^{\top}$ which was already reported to use elemental sulfur as electron acceptor for oxidizing glucose (Ben Hania et al., 2013) was also co-cultured successfully with $D$. vulgaris subsp. vulgaris as confirmed by the increase of the OD at $580 \mathrm{~nm}$ (from 0.08 to 0.19 ) after 23 days of incubation.

The same kind of experiment was performed with the marine Mesotoga genus member, $M$. prima strain MesG1.Ag.4.2 ${ }^{\top}$. However, as glucose was reported to be weakly used by this bacterium, it was replaced by fructose (Nesbø et al., 2012). Similarly to M. prima strain PhosAc3, no growth was observed in fructose/sulfate medium and only a poor growth was obtained in the presence of $\mathrm{S}^{\circ}$ (Fig. 2B). When M. prima strain MesG1.Ag.4.2 $2^{\top}$ was cocultured with Desulfovibrio salexigens which was isolated from a marine environment as well, the OD at $580 \mathrm{~nm}$ increased during the first 9 days to reach around 0.4 $\mathrm{OD}_{580 \mathrm{~nm}}$ unit, thus demonstrating the establishment of the co-culture when growing on fructose. At that time, around $13 \mathrm{mM}$ acetate and $14 \mathrm{mM} \mathrm{H}_{2} \mathrm{~S}$ were produced (Table 1). A lower amount of acetate was produced in mono-culture in the presence of $\mathrm{S}^{\circ}$, in agreement with a lower growth under this condition (Table 1, Fig. 2B). Whatever the growth condition, the measured hydrogen concentration in the gas phase was around $1 \mu \mathrm{M}$.

To ensure that both $M$. prima strain PhosAc3 and $D$. vulgaris subsp. vulgaris actually grew under co-culture conditions, each cell-type was counted by epifluorescence microscopy after DAPI staining. This was made possible since the two bacteria have distinct cell morphology, easily 


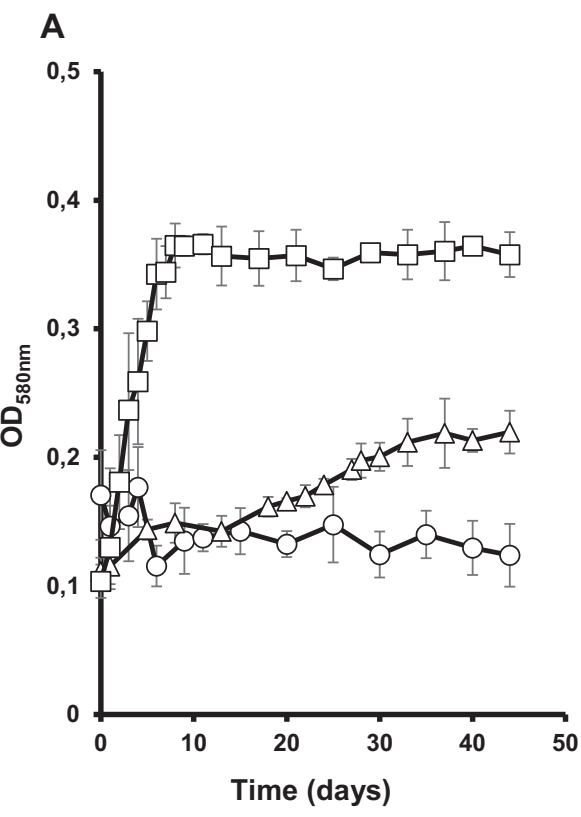

recognizable: small vibrios for $D$. vulgaris and pleomorphic rods surrounded with the sheath-like structure known as toga for M. prima strain PhosAc3 (Fig. 3). It revealed that four and five times more $M$. prima strain PhosAc3 and $D$. vulgaris cells, respectively, were present after 10 days of incubation in comparison to the inoculation time (time 0). At time 0 , a Mesotoga/Desulfovibrio cell number ratio of $5.16 \pm 1.06$ was counted. This ratio changed very little during the growth time $(5.54 \pm 1.11,3.97 \pm 0.5$ and $3.72 \pm 0.91$ after 5,10 and 20 days of incubation respectively), showing that in a well-established co-culture, Mesotoga cells were about four times more abundant than Desulfovibrio cells.

Our data contrast with that previously obtained on M. prima strain MesG1.Ag4. $2^{\top}$ which has been described as a sugar fermentative bacterium producing acetate as
Fig. 2. A. Growth curves of $M$. prima strain PhosAc3 (triangle) and $D$. vulgaris (circle) in pure culture or in co-culture (square) in $20 \mathrm{mM}$ glucose/ $28 \mathrm{mM}$ sulfate containing medium. B. Growth curves of $M$. prima strain MesG1.A4.2 $2^{\top}$ in the presence (triangle) or the absence (circle) of $\mathrm{S}^{\circ}$ and in coculture with $D$. salexigens (square) in $20 \mathrm{mM}$ fructose/28 mM sulfate medium
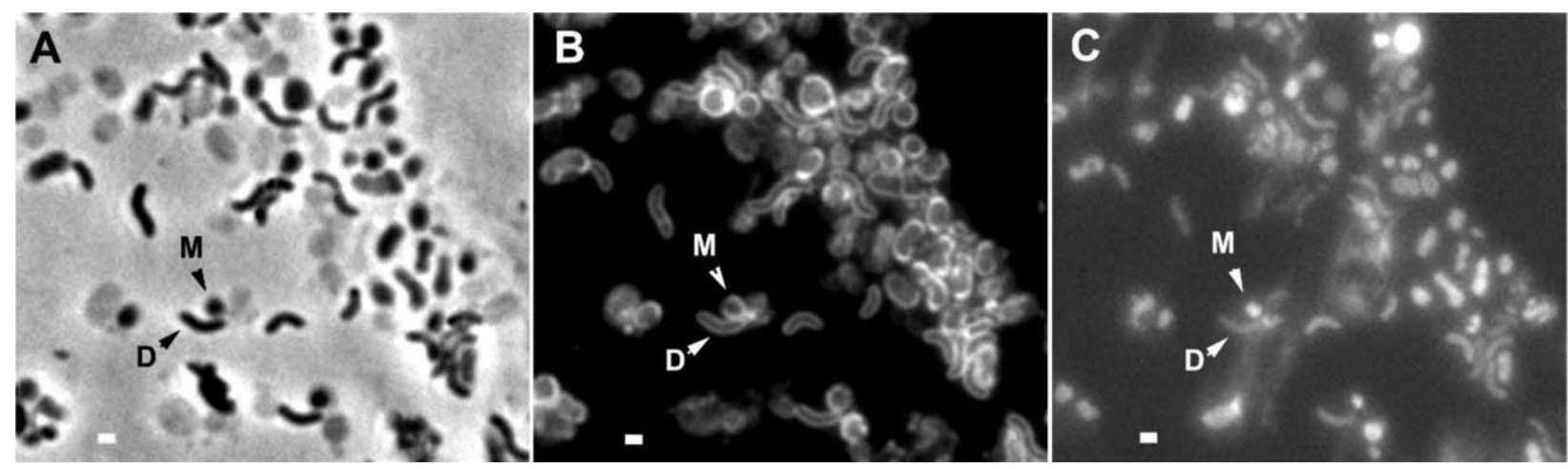

Fig. 3. Microscopy images of $D$. vulgaris and $M$. prima strain PhosAc3 in co-culture.

Exponentially growing co-culture was confined between a coverslip and a thin layer of solid medium culture in a custom hermetic chamber for anaerobic observation. (A) Phase contrast images (DIA), (B) membrane staining (FM4-64) and (C) nucleoid staining (DAPI). Scale bar = 1 $\mu \mathrm{m}$. $\mathrm{M}$ and D arrows show Mesotoga prima strain PhosAc3 and Desulfovibrio vulgaris subsp. vulgaris respectively. 
Under the growth condition used for the co-cultures (sugar/sulfate), Desulfovibrio spp. were unable to grow. This indicated that, in the co-cultures, Desulfovibrio spp. required metabolite(s) or end-product(s) of sugar metabolism released by Mesotoga strains. To test this hypothesis and to check whether cell-to-cell contacts were required, $M$. prima strain PhosAc3 and D. vulgaris subsp. vulgaris were co-cultured using a special set-up where the two bacteria were grown into two compartments separated with a dialysis membrane (Supporting Information Fig. S1). Under these conditions, after 18 days of incubation at $37^{\circ} \mathrm{C}$, the $\mathrm{OD}_{580 \mathrm{~nm}}$ increased from $0.02 \pm 0.01$ to $0.31 \pm 0.03$ in the $M$. prima compartment and from $0.07 \pm 0.01$ to $0.34 \pm 0.03$ in the $D$. vulgaris compartment. This clearly indicated that in co-cultures, both bacteria species were able to grow without the requirement of any cellto-cell contact between them and suggested that Mesotoga and Desulfovibrio exchanged metabolites, required for their respective growth, able to diffuse across a dialysis membrane. Several attempts to co-culture Mesotoga with hydrogenotrophic methanogens as $\mathrm{H}_{2}$ scavenger (Methanospirillum hungatei, Methanobacterium congolense or Methanobacterium aarhusense) were only successful using M. hungatei; after 67 days of incubation at $37^{\circ} \mathrm{C}$, increase of the $\mathrm{OD}_{580 \mathrm{~nm}}(0.135 \pm 0.03)$ and microscopic observations (data not shown) indicated a growth of both microorganisms while no growth was observed when only one partner was present in the culture medium.

All the data presented above suggested that $M$. prima strain PhosAc3 and $D$. vulgaris growths were tightly coupled through a mutualistic/syntrophic association. Several examples of syntrophic associations involving the exchange of hydrogen or formate between the partners have been documented (Stams and Plugge, 2009). To test the involvement of hydrogen as a possible metabolic intermediate, Desulfovibrio and Mesotoga were cultured in two separate serum bottles whose headspaces communicated via tubing (Supporting Information Fig. S1), allowing only gas to be exchanged. After 24 days of incubation at $37^{\circ} \mathrm{C}$, the $\mathrm{OD}_{580 \mathrm{~nm}}$ increased from $0.02 \pm 0.01$ to $0.24 \pm 0.03$ in the $M$. prima compartment, and from $0.06 \pm 0.01$ to $0.22 \pm 0.02$ in the $D$. vulgaris compartment. These results indicated that (a) gaseous substance(s) exchanged between the two partners permitted syntrophic growth between Mesotoga and Desulfovibrio. Given the previous examples of syntrophic associations, our knowledge of the metabolism of $M$. prima and $D$. vulgaris and the fact that we did detect only traces of $\mathrm{H}_{2}$ but not that of formate, we suggest that the best candidate as exchanged metabolite was hydrogen. As hydrogen metabolism requires hydrogenases, hydrogenase activity and glucose-dependent $\mathrm{H}_{2}$ production from M. prima strain PhosAc3 cells were thus tested.

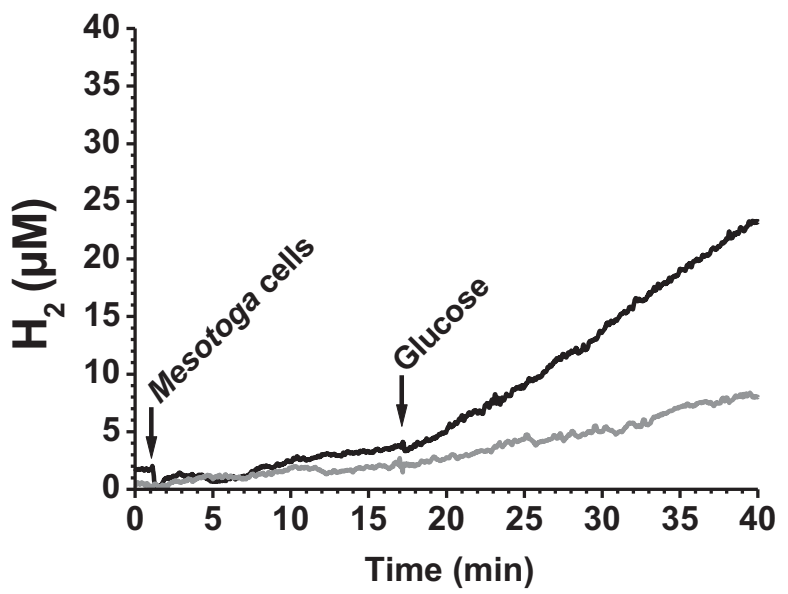

Fig. 4. Hydrogen production by M. prima strain PhosAc3 cells. Hydrogen evolution was measured on Mesotoga whole washed cells grown either in dialysis tubing co-cultured with Desulfovibrio vulgaris subsp. vulgaris in glucose/sulfate medium (black line) or grown in pure culture on glucose in the presence of elemental sulfur (grey line). Experiments were carried out in a respirometry chamber $(1.9 \mathrm{ml})$ in Tris- $\mathrm{HCl} 0.1 \mathrm{M} \mathrm{NaCl} 0.15 \mathrm{M}$ buffer $(\mathrm{pH} 7.5)$ at $37^{\circ} \mathrm{C}$ using a specific $\mathrm{H}_{2}$ microsensor. Addition of cells (1.6 mg total protein for both samples) and glucose (50 $\mathrm{mM}$ final concentration) are indicated by arrows.

Hydrogenase activity and hydrogen production in M. prima strain PhosAc3

Glucose-dependent hydrogen production by $M$. prima strain PhosAc3, was evaluated using a Clark-type hydrogen microsensor on concentrated cells from either pure culture in the presence of elemental sulfur or co-culture with $D$. vulgaris in dialysis membrane-separated compartments (Fig. 4). A hydrogen production of $1.13 \pm 0.25 \mathrm{nmol}$ $\mathrm{H}_{2} / \mathrm{min} / \mathrm{mg}$ protein using glucose as substrate was measured by M. prima strain PhosAc3 when co-cultured with D. vulgaris while only $0.42 \pm 0.06 \mathrm{nmol} \mathrm{H}_{2} / \mathrm{min} / \mathrm{mg}$ protein were produced when it was cultured alone in the presence of $\mathrm{S}^{\circ}$.

Spectrophotometric hydrogenase activity assays using methyl-viologen revealed hydrogenase activities of $33 \pm 3$ $\mathrm{nmol} \mathrm{H}_{2}$ produced $/ \mathrm{min} / \mathrm{mg}$ total protein and $5920 \pm 930$ $\mathrm{nmol} \mathrm{H}_{2}$ consumed $/ \mathrm{min} / \mathrm{mg}$ total protein when M. prima strain PhosAc3 was co-cultured with $D$. vulgaris while no hydrogenase activity could be detected when it was cultured alone in the presence of elemental sulfur. These data showed that hydrogen production and hydrogenase activity were higher when $M$. prima was cultured in the presence of $D$. vulgaris than in its absence, suggesting that $M$. prima was able to regulate the amount of hydrogenase in the cells, depending on the presence of a $\mathrm{H}_{2}$ scavenger such as $D$. vulgaris.

Overall, these experimental data clearly showed that $M$. prima strain PhosAc3 was able to oxidize efficiently glucose through an interspecies $\mathrm{H}_{2}$ transfer with $D$. vulgaris, 
Table 2. Enzymes occurrence in Thermotogae.

\begin{tabular}{|c|c|c|c|c|}
\hline & $\begin{array}{l}\text { Bifurcating } \\
{[\mathrm{FeFe}] \text { hydrogenase }}\end{array}$ & $\begin{array}{l}\text { Fd-dependent } \\
{[\text { FeFe] hydrogenase }}\end{array}$ & $\begin{array}{l}\mathrm{Na}+-\mathrm{NQR} \\
\text { complex }\end{array}$ & Rnf complex \\
\hline Fervidobacterium nodosum Rt17-B1 & + & + & + & $+/$ Short RnfB \\
\hline Fervidobacterium pennivorans DSM 9078 & + & + & + & +/Short RnfB \\
\hline Pseudothermotoga thermarum DSM 5069 & + & + & + & +/Short RnfB \\
\hline Pseudothermotoga lettingae TMO & + & + & + & +/Short RnfB \\
\hline Thermosipho africanus TCF52B & + & + & + & +/Short RnfB \\
\hline Thermosipho melanesiensis BI429 & + & + & + & +/Short RnfB \\
\hline Thermotoga neapolitana DSM 4359 & + & + & - & +/Short RnfB \\
\hline Thermotoga maritima MSB8 & + & + & - & +/Short RnfB \\
\hline Thermotoga petrophila RKU-1 & + & + & - & $+/$ Short RnfB \\
\hline Thermotoga naphthophila RKU-10 & + & + & - & +/Short RnfB \\
\hline Marinitoga piezophila KA3 & - & + & - & +/Short RnfB \\
\hline Petrotoga mobilis SJ95 & - & + & + & +/Short RnfB \\
\hline Kosmotoga olearia TBF 19.5 .1 & - & + & - & +/Long RnfB \\
\hline Mesotoga infera VNs100 & - & + & + & +/Long RnfB \\
\hline Mesotoga prima MesG1 Ag 42 & - & + & + & +/Long RnfB \\
\hline Mesotoga prima PhosAc3 & - & + & + & +/Long RnfB \\
\hline
\end{tabular}

the latter acting as a hydrogen scavenger. It is thus capable of syntrophic association with Desulfovibrio that leads to a more efficient growth, as evidenced by a significant improvement of biomass production and growth rate, than when cultured alone in the presence of elemental sulfur as terminal electron acceptor.

\section{Mesotoga spp. genome mining regarding hydrogen} production and sugar oxidation

Because hydrogen metabolism is a key pathway for this syntrophic association, the occurrence of genes encoding hydrogenases in the M. prima strain PhosAc3 genome was explored. No blast hits were found when using [NiFe]hydrogenase sequences as input (data not shown), while one [FeFe]-hydrogenase homologue was detected in $M$. prima strain PhosAc3 (MESO_PHOSAC3V1_90124). Close homologues were found in the two other Mesotoga members (i.e. $M$. prima strain MesG1.Ag4.2 ${ }^{\top}$ (Theba_0443) and M. infera strain VNs100 (MESINFAv2 2088)). The phylogenetic analysis of Thermotogae [FeFe]hydrogenases showed that the sequences of Mesotoga branched specifically with the ferredoxin-dependent [FeFe]-hydrogenase TM0201 of Thermotoga maritima strain MSB8 (Supporting Information Fig. S2). Interestingly, no homologue of the bifurcating trimeric [FeFe]hydrogenase (TM1424-1426) from T. maritima strain MSB8 (Schut and Adams, 2009) was found in any Mesotoga strains so far sequenced (Table 2). Bifurcating [FeFe]hydrogenases play an important function in the link between the carbohydrates oxidation via a classical Embden-Meyerhof pathway and the production of hydrogen in T. maritima by using the exergonic oxidation of ferredoxin to drive the endergonic oxidation of NADH to produce $\mathrm{H}_{2}$ (Schut and Adams, 2009; Buckel and Thauer,
2013). It thus provides a mechanism to regenerate NAD from $\mathrm{NADH}$ by producing $\mathrm{H}_{2}$, even if this reaction is thermodynamically unfavourable. In contrast with $T$. maritima, M. prima strain PhosAc3 is unable to ferment glucose; we propose that this metabolic difference is due to the absence of the bifurcating [FeFe]-hydrogenase in M. prima preventing the efficient re-oxidation by this enzyme of NADH generated by the NAD-dependent glyceraldehyde 3-phosphate dehydrogenase during glucose oxidation.

A search in M. prima strain PhosAc3 genome revealed the presence of two candidates for NADH re-oxidation: the $\mathrm{Na}^{+}$-translocating $\mathrm{NADH}$-quinone oxidoreductase $\left(\mathrm{Na}^{+}-\mathrm{NQR}\right)$ complex (MESO_PHOSAC3V1_90501-MESO_PHOSAC3V1_90504) and the type 2 Rnf complex (MESO_PHOSAC3V1_120288-MESO_PHOSAC3V1_ 120293). It has been proposed that both $\mathrm{Na}^{+}-\mathrm{NQR}$ and Rnf complexes would allow the re-oxidation of the NADH by transferring electrons to quinone and ferredoxin, coupled to $\mathrm{Na}^{+}$and $\mathrm{Na}^{+} / \mathrm{H}^{+}$translocation through the membrane respectively (Hayashi et al., 2001; Biegel and Müller, 2010; Biegel et al., 2011; Barquera, 2014). While the Rnf complex of type 2 is present in all Thermotogae, the $\mathrm{Na}^{+}$-NQR complex is absent in Kosmotoga olearia, Marinitoga piezophila and Thermotoga species (Table 2). Phylogenetic analysis of the genes encoding Rnf complex of type 2 indicated that this complex was probably present in the ancestor of Thermotogae (Supporting Information Fig. S3). In contrast, the $\mathrm{Na}^{+}-\mathrm{NQR}$ complex is patchy distributed in Thermotogae and the corresponding sequences appeared intermixed with sequences from Spirochaetes, Clostridia and Fusobacteria (Supporting Information Fig. S4), indicating that horizontal gene transfers (HGT) occurred among these lineages and that it could have been secondarily acquired in Thermotogae. Sequences alignment of RnfB subunit from Thermotogae 
revealed that the subunit from $T$. maritima was shorter than that from M. prima strain PhosAc3. This shorter sequence bound only one FeS cluster compared to at least two FeS clusters in the larger ones (Supporting Information Fig. S5). This characteristic was shared by all Thermotogae sequence so far, except members of the Mesotoga and Kosmotoga genera (Supporting Information Fig. S5). Interestingly, the presence of a shorter RnfB subunit correlated very well with the presence of a bifurcating [FeFe] hydrogenase in the organism, suggesting that this peculiar sequence feature could be involved in the specific interaction between the two proteins (Table 2).

\section{Discussion}

M. prima strain PhosAc3 was the first mesophilic cultivated member within the phylum Thermotogae (Ben Hania et al., 2011) which comprised only fermentative thermophilic or hyperthermophilic bacteria (Huber and Hannig 2006; Zhaxybayeva et al., 2009). Here we show that this bacterium is able to consume glucose only in the presence of elemental sulfur, thus clearly indicating that its sugar metabolism is based on an obligatory oxidative pathway, the elemental sulfur being essential to eliminate excess reducing equivalents generated from sugar oxidation. However, because M. prima strain PhosAc3 oxidizes very slowly sugar in the presence of elemental sulfur, one could expect that sulfur reduction is not a true respiratory process linked to oxidative phosphorylation. In this respect, elemental sulfur should serve as electron sink. Nevertheless, further experiments are needed to clarify this peculiar point regarding sulfur reduction. It is noteworthy that hydrogen was detected only as traces (around $1 \mu \mathrm{M}$ in the gas phase) in any tested growth condition. The same type of sugar metabolism was demonstrated for M. prima strain MesG1.Ag. $4.2^{\top}$ and $M$. infera strain VNs $100^{\top}$, the two other known members of the Mesotoga genus so far. All these data contrast with energy metabolism of thermophilic and hyperthermophilic Thermotogae known to (i) ferment sugars with substantial production of hydrogen (over $1 \mathrm{mM}$ ) (Schut and Adams, 2009; Cappelletti et al., 2014) and (ii) reduce elemental sulfur facultatively (Huber and Hannif, 2006; Cappelletti et al., 2014). Strictly following the definition of fermentation as 'an energy yielding process in which an energy substrate is metabolized without the involvement of an exogenous electron acceptor' (Singleton and Sainsbury, 2001), Mesotoga spp. should not be considered as sugar-fermenting but rather as sugar-oxidizing microorganisms.

We demonstrate also that $M$. prima strain PhosAc3, M. prima strain MesG1.Ag.4. $2^{\top}$ and $M$. infera strain VNs $100^{\top}$ grow more efficiently in syntrophic association with a hydrogenotrophic SRB, which serves as a terminal biological electron acceptor, than as pure culture in the presence of sulfur. Our data strongly suggest the existence of an interspecies hydrogen transfer between these Mesotoga species and hydrogenotrophic SRB. It should be noted that while we succeeded to co-culture $M$. prima strain PhosAc3 with all hydrogenotrophic SRB tested, attempts to co-culture it with hydrogenotrophic methanogens were only successful with $M$. hungatei only after several months of incubation. Because sulfate-reducing bacteria are known to have higher affinity for hydrogen than methanogens (Kristjansson et al., 1982; Robinson and Tiedje 1984), it may indicate that the oxidation of substrates by Mesotoga requires a very low partial hydrogen pressure that could be better established by SRB than methanogens, with the exception of $M$. hungatei which is known to have the highest affinity for $\mathrm{H}_{2}$ among methanogens (Robinson and Tiedje, 1984). In this respect, here we provide evidence for Thermotogae members to perform an efficient obligatory sugar oxidation through a syntrophic association with a hydrogenotrophic microbial partner.

We propose that the metabolic difference between Thermotoga spp. and M. prima strain PhosAc3 regarding sugar degradation is related to the absence of a bifurcating [FeFe]-hydrogenase in the latter, preventing it to ferment sugar. In contrast with $T$. maritima, the absence of this enzyme in $M$. prima would prevent the efficient re-oxidation of $\mathrm{NADH}$, generated during glucose oxidation, linked to $\mathrm{H}_{2}$ production. In the absence of an external electron acceptor, NADH accumulation would thus lead to growth inhibition. During the syntrophic association with a hydrogenotrophic partner, the ferredoxin-dependent [FeFe]hydrogenase (locus tag MESO_PHOSAC3V1_90124) would produce hydrogen that would be, in turn, metabolized by the hydrogenotrophic partner. It has been proposed that the Rnf complex in T. maritima is involved in maintaining the appropriate ferredoxin/NADH ratio for the bifurcating [FeFe]-hydrogenase and other cell processes (Schut and Adams, 2009). Similarly, we advance that the M. prima strain PhosAc3 Rnf complex (MESO_PHOSAC3V1_120288-MESO_PHOSAC3V1_120293) would participate in hydrogen metabolism by driving reverse electron flow from $\mathrm{NADH}$, generated by glucose oxidation, to the reduction of ferredoxin which then delivers electrons to the ferredoxin-dependent $[\mathrm{FeFe}]$-hydrogenase. The $\mathrm{Na}^{+}$NQR complex (MESO_PHOSAC3V1_90501-MESO_ PHOSAC3V1_90504) would also participate in re-oxidation of the reduced NADH generated from glucose oxidation.

Because M. prima strain PhosAc3, M. prima strain MesG1.Ag4. $2^{\top}$ and $M$. infera strain $\mathrm{VNs} 100^{\top}$ are all able to oxidize sugars, one can hypothesize that this capacity was inherited from their common ancestor. Actually, like $M$. prima strain PhosAc3, M. prima strain MesG1.Ag4. $2^{\top}$ genome encodes homologous ferredoxin-dependent [FeFe]-hydrogenase (Theba_0443) as well as $\mathrm{Na}^{+}-\mathrm{NQR}$ 
(Theba_0829-Theba_0833) and Rnf (Theba_1343Theba_1348) complexes but no bifurcating [FeFe]-hydrogenase. We thus propose that the efficient sugar-oxidizing metabolism linked to a syntrophic association with a hydrogenotrophic bacterium can be extrapolated to all members of the Mesotoga genus.

The importance of obligate interspecies hydrogen transfer for substrate oxidation has been documented in many occasions with a peculiar emphasis for fatty acids, ethanol as well as aromatic and alicyclic compounds (Sieber et al., 2014; Schmidt et al., 2016). In contrast, such process involving a hydrogenotrophic partner to degrade easily fermentable substrates as carbohydrates has been reported only a few times, as for Syntrophococcus sucromutans, isolated from rumen of steer (Krumholz and Bryant, 1986) and Bacillus spp., isolated from Lake Constance sediment (Müller et al., 2008). The latter authors, by direct dilution of Lake Constance sediment in mineral agar medium containing Methanosprillum hungatei, provided evidence that obligate sugar-degrading Bacillus sp. using a hydrogenscavenging methanogen as terminal electron acceptor outnumbered those fermenting easily these substrates. These Bacillus spp. were thus believed to be of high ecological significance in these sediments (Müller et al., 2008). The same conclusion may be drawn for $M$. prima strain PhosAc3 since, similarly as for Müller et al. (2008), it has been isolated after high dilution steps from anaerobic digester treating phosphogypsum (Ben Hania et al., 2011; 2015). It may be extended to other Mesotoga strains which have been detected by molecular approaches in various ecosystems including those contaminated by toxic compounds (Nesbø et al., 2006; 2010). Association with hydrogenotrophic SRB and possibly with high hydrogen affinity hydrogenotrophic methanogens such as $M$. hungatei would thus be of primary ecological importance for growth of Mesotoga spp. and other obligate-oxidizing bacteria in their natural habitats. Interestingly, all these bacteria (S. sucromutans, Bacillus and Mesotoga spp.) produce acetate as the sole organic acid end-product of sugar metabolism. Their ecological role as saccharolytic acetogenic bacteria in association with anaerobic hydrogen scavengers (e.g. sulfate-reducing bacteria), might have been underestimated so far. It thus deserves further investigations especially in sugar containing environments, to enlarge the current view of the microbiology of anaerobic digestion of organic matter (e.g. carbohydrates) that typically gives a predominant role to classical sugar-fermenting bacteria over syntrophic sugar-oxidizing associations.

\section{Experimental procedures}

\section{Media and culture conditions}

M. prima strain PhosAc3 and M. infera strain $\mathrm{VNs}_{100^{\top}}$ were grown with glucose $(20 \mathrm{mM})$ as carbon and energy sources at $37^{\circ} \mathrm{C}$ in medium containing per litre $0.3 \mathrm{~g} \mathrm{KH}_{2} \mathrm{PO}_{4} ; 0.3 \mathrm{~g}$ $\mathrm{K}_{2} \mathrm{HPO}_{4} ; 1.0 \mathrm{~g} \mathrm{NH} \mathrm{Cl}_{4} ; 2.0 \mathrm{~g} \mathrm{NaCl} ; 0.1 \mathrm{~g} \mathrm{KCl} ; 0.1 \mathrm{~g}$ $\mathrm{CaCl}_{2} .2 \mathrm{H}_{2} \mathrm{O} ; 0.5 \mathrm{~g} \mathrm{MgCl}_{2} .6 \mathrm{H}_{2} \mathrm{O} ; 1 \mathrm{~g}$ yeast extract; $0.5 \mathrm{~g}$ cysteine-HCl; $0.16 \mathrm{~g}$ sodium acetate; $1 \mathrm{ml}$ Widdel trace element solution (Widdel and Pfennig, 1981) and $1 \mathrm{ml}$ resazurin $0.1 \%$. When specified, $10 \mathrm{~g}$ of elemental sulfur $\left(\mathrm{S}^{0}\right)$ per litre of medium was added. Before culture inoculation, $0.2 \mathrm{ml}$ of $10 \%$ (wt/vol) $\mathrm{NaHCO}_{3}, 0.1 \mathrm{ml}$ of $2 \%$ (wt/vol) $\mathrm{Na}_{2} \mathrm{~S}-9 \mathrm{H}_{2} \mathrm{O}$ and glucose were injected from sterile stock solutions in the culture medium. Mesotoga prima strain MesG1.Ag4.2 ${ }^{\top}$ was grown in the same culture medium mentioned above containing fructose $(20 \mathrm{mM})$ instead of glucose in the presence of $25 \mathrm{~g} \mathrm{I}^{-1}$ $\mathrm{NaCl}$. Desulfovibrio vulgaris subsp. vulgaris, $D$. salexigens and $D$. gibsoniae were grown in the same culture medium as for $M$. prima strain PhosAc3 with the sugar being replaced by hydrogen $\left(\mathrm{H}_{2}: \mathrm{CO}_{2} ; 80: 20 \mathrm{vol} / \mathrm{vol}\right)$ as electron donor and elemental sulfur by sodium sulfate $\left(\mathrm{Na}_{2} \mathrm{SO}_{4}\right)\left(4 \mathrm{~g} \mathrm{I}^{-1}\right)$ as electron acceptor. Twenty-five grams per litre $\mathrm{NaCl}$ was added to the culture medium for growth of $D$. salexigens. Methanospirillum hungatei, $M$. congolense and $M$. aarhusense, were grown in the same culture medium as for sulfate-reducing bacteria but without sulfate. Co-cultures were performed in the same medium as for culturing Mesotoga species alone but without elemental sulfur that was replaced by sodium sulfate $\left(4 \mathrm{~g} \mathrm{I}^{-1}\right)$ in the case of co-cultures with sulfate-reducing bacteria.

Cultures and co-cultures were performed under anaerobic conditions in Hungate tubes or in large volume flasks. Cocultures were established by mixing (10\% vol/vol each) exponentially growing (i) Mesotoga strain PhosAc3 and either $D$. vulgaris subsp. vulgaris, D. gibsoniae, M. hungatei, M. congolense or $M$. aarhusense, (ii) $M$. infera strain $\mathrm{VNs}^{100^{\top} \text { and }}$ D. vulgaris subsp. vulgaris, (iii) M. prima strain MesG1.Ag4. $2^{\top}$ and $D$. salexigens. Fresh medium was inoculated with $10 \%$ ( $\mathrm{vol} / \mathrm{vol}$ ) of exponentially growing cells and incubated at $37^{\circ} \mathrm{C}$. Growth was monitored by measurement of the OD at $580 \mathrm{~nm}$ of the culture directly in the Hungate tube. Growth curves and metabolites quantification were obtained from at least 2 independent replicates.

\section{Analytical methods}

Soluble sulfides were quantified according to the Cord-Ruwish (1985) method by using a Shimadzu UV-160A spectrophotometer (Shimadzu, Kyoto, Japan). Sugars (lactose, glucose), organic acids (acetate, lactate, propionate, butyrate, formate) and ethanol determination was carried out by high performance liquid chromatography (HPLC) as described by Fardeau et al. (1997). For hydrogen quantification, $1 \mathrm{ml}$ of culture headspace sample (gas phase) was injected into a Shimadzu 8A TCD-GC system (Shimadzu, Kyoto, Japan) equipped with a concentric CTR1 column (Alltech, Columbia, MD, USA), connected to a computer running WINILAB III software (Perichrom, Saulx les Chartreux, France) (Fardeau et al., 1997). Unless otherwise indicated, analytical measures were performed on duplicate culture tubes or bottles.

\section{Cells counting by microscopy}

For preparing the microscope observation chamber, all steps were performed in an anaerobic chamber (Coy Laboratory, 
Grass Lake, MI, USA). One hundred microlitre of the M. prima strain PhosAc3 and $D$. vulgaris subsp. vulgaris co-culture were centrifuged for $3 \mathrm{~min}$ at $10000 \mathrm{~g}$. DAPI staining was performed by resuspending the cells pellet in $100 \mu \mathrm{l}$ of $10 \mathrm{mM}$ Tris- $\mathrm{HCl}(\mathrm{pH} 7.6), 8 \mathrm{mM} \mathrm{MgSO}{ }_{4}$ and $1 \mathrm{mM} \mathrm{KH}_{2} \mathrm{PO}_{4}$ buffer (TPM buffer) containing $5 \mathrm{ng} \mathrm{\mu l}^{-1}$ of $4^{\prime}, 6$-diamidino-2-phenylindole (DAPI). After 20 min of incubation in the dark, cells were washed three times with TPM buffer. The DNA was stained under anaerobic conditions to limit the exposure of the cells to air. The cells were placed between a glass coverslip and a thin layer of LS4D-YE medium supplemented with $10 \mathrm{ng} \mathrm{Il}^{-1}$ of FM4-64® (Invitrogen) and $1.5 \%$ (wt/vol) of Phytage ${ }^{\mathrm{TM}}$ (Sigma-Aldrich) that was previously prepared under anaerobic conditions. The preparation was then transferred in a hermetic microscope observation chamber (Fievet et al., 2015) and put into a standard temperature-controlled inverted microscope. Pictures were acquired with a Nikon TiE-PFS inverted epifluorescence microscope, $100 \times$ NA1.3 oil PhC objective (Nikon) and Hamamatsu Orca-R2 camera. Image processing was controlled by the NIS-Element software (Nikon).

\section{Hydrogenase activity measurements by spectrophotometry}

Pure cultures of $M$. prima strain PhosAc3 (1 I) were grown in the presence of elemental sulfur until the $\mathrm{OD}_{580 \mathrm{~nm}}$ reached 0.28 . Cells were harvested by centrifugation ( $5000 \mathrm{~g}$ for 30 $\mathrm{min}$ ), rinsed once with $50 \mathrm{ml}$ of $50 \mathrm{mM}$ Tris- $\mathrm{HCl}, \mathrm{NaCl} 200 \mathrm{mM}$ (pH 7.5) buffer and finally resuspended in $500 \mu \mathrm{l}$ of $100 \mathrm{mM}$ HEPES buffer (pH 8.0). When M. prima strain PhosAc3 was co-cultured with $D$. vulgaris subsp. vulgaris in separated compartments, cells were prepared as above from $500 \mathrm{ml}$ of culture. For hydrogenase uptake activities, a rubber-stopper sealed cuvette containing $1 \mathrm{ml}$ of $100 \mathrm{mM}$ HEPES buffer $(\mathrm{pH}$ 8.0), $75 \mu \mathrm{l}$ of $100 \mathrm{mM}$ methyl-viologen was bubbled for $5 \mathrm{~min}$ with hydrogen. Then, a defined amount of cells suspension (from 10 to $50 \mu \mathrm{l}$ ) and $5 \mu \mathrm{l}$ of $1 / 10$ Triton X100 diluted in HEPES buffer was added. The OD at $604 \mathrm{~nm}$ was recorded over the time. As controls, methyl-viologen reduction was followed in cuvettes without hydrogen bubbling and without addition of cells.

For $\mathrm{H}_{2}$ production activity, a rubber-stopper sealed cuvette containing $1 \mathrm{ml}$ of $100 \mathrm{mM}$ HEPES buffer ( $\mathrm{pH}$ 8.0) was bubbled for 5 min with Argon. A defined amount of cells suspension (from 10 to $50 \mu \mathrm{l}$ ) was then added and the cuvette was bubbled with Argon for 2 min longer. Then, $100 \mu$ of dithionite-reduced methyl-viologen $(10 \mathrm{mM})$ and $5 \mu \mathrm{l}$ of $1 / 10$ Triton X100 diluted in HEPES buffer were added. The decrease of the OD at $604 \mathrm{~nm}$ was recorded over the time. The auto-oxidation kinetic of reduced methyl-viologen was measured in cuvette in the absence of cells. $\mathrm{H}_{2}$ production activity was obtained by subtracting the auto-oxidation activity from the methy-viologen oxidation activity in the presence of cells.

All buffers, reactives and cells suspensions were kept under anaerobic conditions and continuously flushed with Argon.

\section{$\mathrm{H}_{2}$ production monitoring by Clark-based microsensor}

Hydrogen production was also measured on $M$. prima strain PhosAc3 cells grown on glucose either as mono-culture in the presence of elemental sulfur or as co-culture with $D$. vulgaris in dialysis membrane-separated compartments as described in Supporting Information Fig. S1. Cells were harvested at the end of the exponential phase and washed three times with an anaerobic $0.1 \mathrm{M}$ Tris- $\mathrm{HCl}, 0.15 \mathrm{M} \mathrm{NaCl}(\mathrm{pH} 7.5)$ buffer saturated with $\mathrm{N}_{2}$. The measurements were carried out in a $1.9 \mathrm{ml}$ sealed anaerobic cuvette filled with $0.1 \mathrm{M}$ Tris- $\mathrm{HCl}, 0.15 \mathrm{M}$ $\mathrm{NaCl}(\mathrm{pH} 7.5)$ buffer equilibrated at $37^{\circ} \mathrm{C}$ and containing cells suspension. After $15 \mathrm{~min}, 50 \mathrm{mM}$ glucose (final concentration) was added and $\mathrm{H}_{2}$ production was monitored with a $\mathrm{H}_{2}-\mathrm{MR}$ $500 \mu \mathrm{m}$ microsensor plugged to a Microsensor Multimeter (Unisense, Aarhus, Denmark). Electrode was calibrated with known amounts of pure $\mathrm{H}_{2}$ gas saturated in water at $37^{\circ} \mathrm{C}$ $(750 \mu \mathrm{M})$.

\section{Phylogenetic analyses}

Two thousand seven hundred seventy-five complete prokaryotic proteomes were downloaded at the $\mathrm{NBCl}$ (http://www. ncbi.nlm.nih.gov/). Only one representative proteome per species was kept for analyses. The 1369 corresponding proteomes (including 14 Thermotogae species, Supporting Information Table S1) were gathered to build a local database. This database was queried with BLASTP (v2.2.26) (Altschul et al., 1990) to identify homologues of the studied proteins. Mesotoga prima strain PhosAc3 or T. maritima strain MSB8 protein sequences were used as seed. The 60 protein sequences displaying the highest scores and E-values lower than $10^{-3}$ were retrieved. Homologues from $M$. prima strain PhosAc3 and $M$. infera strain $\mathrm{VNs} 100^{\top}$ were downloaded from the MAGE platform at the genoscope (https://www.genoscope.cns.fr). The retrieved homologues were aligned with MAFFT v7 (option L-INS-I) (Katoh and Standley, 2013). The resulting multiple alignments were visually inspected with SEAVIEW v.4.5.4 (Gouy et al., 2010) and trimmed with BMGE v1.1 (Criscuolo and Gribaldo, 2010).

Maximum likelihood phylogenetic trees were inferred with PHYML v.3.1 (Guindon et al., 2010) implemented in the SEAVIEW program (Gouy et al., 2010) using the 'Le and Gascuel evolutionary model' (Le and Gascuel, 2008) and a gamma distribution with four categories of sites to take into account the heterogeneity of site evolutionary rates. The $\mathrm{NNI}+$ SPR option was used to explore tree topologies. The robustness of the resulting trees was evaluated using the non-parametric bootstrap procedure implemented in PHYML (100 replicates of the original dataset).

\section{Acknowledgements}

The authors are grateful to Alison Strutt for revising the English text and to Matthieu Robbino for participating in $\mathrm{H}_{2}$ production measurements. The authors do not have any conflict of interest to declare.

\section{References}

Altschul, S.F., Gish, W., Miller, W., Myers, E.W., and Lipman, D.J. (1990) Basic local alignment search tool. J Mol Microbiol 215: 403-410.

Antoine, E., Cilia, V., Meunier, J.R., Guezennec, J., Lesongeur, F., and Barbier, G. (1997) Thermosipho 
melanesiensis sp. nov., a new thermophilic anaerobic bacterium belonging to the order Thermotogales, isolated from deep-sea hydrothermal vents in the southwestern Pacific Ocean. Int J Syst Bacteriol 47: 1118-1123.

Barquera, B. (2014) The sodium pumping NADH: quinone oxidoreductase (Na+-NQR), a unique redox-driven ion pump. J Bioenerg Biomembr 46: 289-298.

Ben Hania, W., Ghodbane, R., Postec, A., Brochier-Armanet, C., Hamdi, M., and Fardeau, M.L. (2011) Cultivation of the first mesophilic representative ("mesotoga") within the order Thermotogales. Syst Appl Microbiol 34: 581-585.

Ben Hania, W., Godbane, R., Postec, A., Hamdi, M., Ollivier, B., and Fardeau, M.-L. (2012) Defluviitoga tunisiensis gen. nov., sp. nov., a thermophilic bacterium isolated from a mesothermic and anaerobic whey digester. Int J Syst Evol Microbiol 62: 1377-1382.

Ben Hania, W., Postec, A., Aüllo, T., Ranchou-Peyruse, A., Erauso, G., Brochier-Armanet, C., et al. (2013) Mesotoga infera sp. nov., a novel mesophilic member of the order Thermotogales, isolated from an underground gas storage in France. Int J Syst Evol Microbiol 63: 3003-3008.

Ben Hania, W., Fadhlaoui, K., Brochier-Armanet, C., Persillon, C., Postec, A., et al. (2015) Draft genome sequence of Mesotoga strain PhosAC3, a mesophilic member of the bacterial order Thermotogales, isolated from a digestor treating phosphogypsum in Tunisia. Stand Genomic Sci 10: 12.

Bhandari, V., and Gupta, R.S. (2014) Molecular signatures for the phylum (class) Thermotogae and a proposal for its division into three orders (Thermotogales, Kosmotogales ord. nov. and Petrotogales ord. nov.) containing four families (Thermotogaceae, Fervidobacteriaceae fam. nov., Kosmotogaceae fam. nov. and Petrotogaceae fam. nov.) and a new genus $P$ seudothermotoga gen. nov. with five new combinations. Antonie van Leeuw 105: 143-168.

Biegel, E., and Müller, V. (2010) Bacterial Na+-translocating ferredoxin: NAD+ oxidoreductase. Proc Natl Acad Sci U S A 107: 18138-18142.

Biegel, E., Schmidt, S., González, J.M., and Müller, V. (2011) Biochemistry, evolution and physiological function of the Rnf complex, a novel ion-motive electron transport complex in prokaryotes. Cell Mol Life Sci 68: 613-634.

Buckel, W., and Thauer, R.K. (2013) Energy conservation via electron bifurcating ferredoxin reduction and proton/ $\mathrm{Na}+$ translocating ferredoxin oxidation. Biochim Biophys Acta 1827: 94-113.

Cappelletti, M., Zannoni, D., Postec, A., and Ollivier, B. (2014). Members of the order Thermotogales: from microbiology to hydrogen production. In Microbial BioEnergy: Hydrogen Production. Zannoni, D., and De Philippis, R. (eds). Springer: Netherlands, pp. 197-224.

Cord-Ruwish, R. (1985) A quick method for the determination of dissolved and precipitated sulfides in culture of sulfate reducing bacteria. J Microbiol Methods 4: 33-36.

Cord-Ruwish, R., Ollivier, B., and Garcia, J.L. (1986) Fructose degradation by Desulfovibrio sp. in pure culture and in coculture with Methanospirillum hungatei. Curr. Microbiol 13: 285-289.

Criscuolo, A., and Gribaldo, S. (2010) BMGE (Block Mapping and Gathering with Entropy): a new software for selection of phylogenetic informative regions from multiple sequence alignments. BMC Evol Biol 10: 210.
Di Pippo, J.L., Nesbo, C.L., Dahle, H., Doolittle, W.F., Birkland, N.K., and Noll, K.M. (2009) Kosmotoga olearia gen. nov., sp. nov., a thermophilic, anaerobic heterotroph isolated from an oil production fluid. Int $J$ Syst Evol Microbiol 59: 2991-3000.

Fardeau, M.L., Ollivier, B., Patel, B.K.C., Magot, M., Thomas, P., Rimbault, A., et al. (1997) Thermotoga hypogea sp. nov., a xylanolytic, thermophilic bacterium from an oil-producing well. Int J Syst Evol Microbiol 47: 1013-1019.

Fievet, A., Ducret, A., Mignot, T., Valette, O., Robert, L., Pardoux, R., et al. (2015) Single-cell analysis of growth and cell division of the anaerobe Desulfovibrio vulgaris Hildenborough. Front Microbiol 8: 1-11.

Gouy, M., Guindon, S., and Gascuel, O. (2010) SeaView version 4: a multiplatform graphical user interface for sequence alignment and phylogenetic tree building. Mol Biol Evol 27: 221-224.

Guindon, S., Dufayard, J.F., Lefort, V., Anisimova, M., Hordijk, W., and Gascuel, O. (2010) New algorithms and methods to estimate maximum-likelihood phylogenies: assessing the performance of PhyML 3.0. Syst Biol 59: 307-321.

Hayashi, M., Nakayama, Y., and Unemoto, T. (2001) Recent progress in the $\mathrm{Na}+$-translocating $\mathrm{NADH}$-quinone reductase from the marine Vibrio alginolyticus. Biochim Biophys Acta 1505: 37-44.

Huber, R., and Hannig, M. (2006). Thermotogales. In The Prokaryotes. Dworkin, M., Falkow, S., Rosenberg, E., Schleifer, K.H., and Stackebrandt, E. (eds). New York: Springer, pp 899-922.

Huber, R., Langworthy, T.A., König, H., Thomm, M., Woese, C.R., Sleytr, U.B., et al. (1986) Thermotoga maritima sp. nov. represents a new genus of unique extremely thermophilic eubacteria growing up to $90^{\circ} \mathrm{C}$. Arch Microbiol 144: 324-333.

Itoh, T., Onishi, M., Kato, S., lino, T., Sakamoto, M., Kudo, T., et al. (2016) Athalassotoga saccharophila gen. nov. sp. nov. isolated from an acidic terrestrial hot spring of Japan, and proposal of Mesoaciditogales ord. nov., Mesoaciditogaceae fam. nov. in the phylum Thermotogae. Int J Syst Evol Microbiol 66: 1045-1051.

Jayasinghearachchi, H.S., and Lal, B. (2011) Oceanotoga teriensis gen. nov., sp. nov., a thermophilic, bacterium isolated from off-shore oil-producing wells. Int J Syst Evol Microbiol 61: 554-560.

Katoh, K., and Standley, D.M. (2013) MAFFT multiple sequence alignment software version 7: improvements in performance and usability. Mol Biol Evol 30: 772-780.

Kristjansson, J.K., Schönheit, P., and Thauer, R.K. (1982) Different Ks values for hydrogen of methanogenic bacteria and sulfate reducing bacteria: an explanation for the apparent inhibition of methanogenesis by sulfate. Arch. Microbiol 131: 278-282.

Krumholz, L.R., and Bryant, M.P. (1986) Syntrophococcus sucromutans sp. nov. gen. nov. uses carbohydrates as electron donors and formate, methoxymonobenzenoids or Methanobrevibacter as electron acceptor systems. Arch Microbiol 143: 313-318.

Le, S.Q., and Gascuel, O. (2008) An improved general amino acid replacement matrix. Mol Biol Evol 25: 1307-1320.

Müller, N., Griffin, B.M., Stingl, U., and Schink, B. (2008) Dominant sugar utilizers in sediment of Lake Constance depend 
on syntrophic cooperation with methanogenic partner organisms. Environ Microbiol 10: 1501-1511.

Muyzer, G., and Stams, A.J. (2008) The ecology and biotechnology of sulphate-reducing bacteria. Nat Rev Microbiol 6: 441-454.

Nesbø, C.L., Dlutek, M., Zhaxybayeva, O., and Doolittle, W.F. (2006) Evidence for existence of "mesotogas", members of the order Thermotogales adapted to low-temperature environments. Appl Environ Microbiol 72: 5061-5068.

Nesbø, C.L., Kumaraswamy, R., Dlutek, M., Doolittle, W.F., and Foght, J. (2010) Searching for mesophilic Thermotogales bacteria: "mesotogas" in the wild. Appl Environ Microbiol 76: 4896-4900.

Nesbø, C.L., Bradnan, D.M., Adebusuyi, A., Dlutek, M., Petrus, A.K., Foght, J., Doolittle, W.F., and Noll, K.M. (2012) Mesotoga prima gen. nov., sp. nov., the first described mesophilic species of the Thermotogales. Extremophiles 16: 387-393.

Reysenbach, A.-L., Liu, Y., Lindgren, A.R., Wagner, I.D., Sislak, C.D., Mets, A., and Schouten, S. (2013) Mesoaciditoga lauensis gen. nov., sp. nov., a moderately thermoacidophilic member of the order Thermotogales from a deepsea hydrothermal vent. Int J Syst Evol Microbiol 63: 47244729.

Robinson, J.A., and Tiedje, J.M. (1984) Competition between sulfate-reducing and methanogenic bacteria for $\mathrm{H}_{2}$ under resting and growing conditions. Arch. Microbiol 137: 26-32.

Schmidt, O., Hink, L., Horn, M.A., and Drake, H. (2016) Peat: home to novel syntrophic species that feed acetate- and hydrogen-scavenging methanogens. ISME $J$ 10: 19541966.

Schut, G.J., and Adams, M.W. (2009) The iron-hydrogenase of Thermotoga maritima utilizes ferredoxin and NADH synergistically: a new perspective on anaerobic hydrogen production. J Bacteriol 191: 4451-4457.

Sieber, J.R., Le, H.M., and Mclnerney, M.J. (2014) The importance of hydrogen and formate transfer for syntrophic fatty, aromatic and alicyclic metabolism. Environ Microbiol 16: 177-188.

Singleton, P., and Sainsbury, D. (2001). Dictionary of Microbiology and Molecular Biology. Chichester: Wiley.

Stams, A.J., and Plugge, C.M. (2009) Electron transfer in syntrophic communities of anaerobic bacteria and archaea. Nat Rev Microbiol 7: 568-577.

Thauer, R.K., Jungermann, K., and Decker, K. (1977) Energy conservation in chemotrophic anaerobic bacteria. Bacteriol Rev 41: 100-180.

Widdel, F., and Pfennig, N. (1981) Studies on dissimilatory sulfate-reducing bacteria that decompose fatty acids. Isolation of new sulfate-reducing bacteria enriched with acetate from saline environments. Description of Desulfobacter postgatei gen. nov., sp. nov. Arch Microbiol 129: 395-400.

Zhaxybayeva, O., Swithers, K.S., Lapierre, P., Fournier, G.P., Bickhart, D.M., DeBoy, R.T., et al. (2009) On the chimeric nature, thermophilic origin, and phylogenetic placement of the Thermotogales. Proc Natl Acad Sci U S A 106: 5865-5870.

Zillig, W., Holz, I., Klenk, H-P., Trent, J., Wunderl, S., Jankovic, D., et al. (1987) Pyrococcus woesei, sp. nov., an ultra-thermophilic marine archaebacterium, representing a novel order, Thermococcales. Syst Appl Microbiol 9: 62-70.

\section{Supporting information}

Additional Supporting Information may be found in the online version of this article at the publisher's web-site:

Fig. S1. (A) Compartment-separated co-culture of M. prima strain PhosAc3 and $D$. vulgaris. $D$. vulgaris was grown inside a dialysis tube (dialysis membrane Spectra/Por, 12$14 \mathrm{~K} \mathrm{MWCO}$ ) that was secluded from the surrounding strain $M$. prima culture by rubber stoppers. The apparatus was incubated in a very slowly moving (30 r.p.m.) rotary shaker at $37^{\circ} \mathrm{C}$. (B) Experimental assembly to grow the co-culture in two different vials linked by a tubing.

Fig. S2. Maximum likelihood phylogeny of the [FeFe] hydrogenases in Thermotogae (101 sequences, 450 amino acid positions kept after trimming of the alignment). Numbers at nodes are bootstrap values (100 replicates of the original dataset). For clarity, values lower than $50 \%$ are not shown. The scale bars indicate the average number of substitutions per site. Colours correspond to taxonomic groups. The complete taxonomy of each strain is indicated on the tree.

Fig. S3. Maximum likelihood phylogenies of the proteins composing the Rnf complex. Numbers at nodes are bootstrap values (100 replicates of the original dataset). For clarity, values lower than $50 \%$ are not shown. The scale bars indicate the average number of substitutions per site. Colours correspond to taxonomic groups. The complete taxonomy of each strain is indicated on the tree. A - RfnCMESO_PHOSAC3v1_120288 (61 sequences, 346 amino acid positions kept after trimming of the alignment). B RfnD-MESO_PHOSAC3v1_120289 (61 sequences, 265 amino acid positions kept after trimming of the alignment). C - RfnG-MESO_PHOSAC3v1_120290 (54 sequences, 75 amino acid positions kept after trimming of the alignment). D - RfnE-MESO_PHOSAC3v1_120291 (62 sequences, 176 amino acid positions kept after trimming of the alignment). E - RfnA-MESO_PHOSAC3v1_120292 (62 sequences, 185 amino acid positions kept after trimming of the alignment). F - RfnB-MESO_PHOSAC3v1_120293 (62 sequences, 107 amino acid positions kept after trimming of the alignment).

Fig. S4. Maximum likelihood phylogenies of the genes composing the $\mathrm{Na}+-\mathrm{NQR}$ complex. Numbers at nodes are bootstrap values (100 replicates of the original dataset). For clarity, values lower than $50 \%$ are not shown. The scale bars indicates the average number of substitutions per site. Colours correspond to taxonomic groups. The complete taxonomy of each strain is indicated on the tree. A - RfnDlike-MESO_PHOSAC3v1_90501 (62 sequences, 215 amino acid positions kept after trimming of the alignment). B NrqC-MESO_PHOSAC3v1_90502 (62 sequences, 86 amino acid positions kept after trimming of the alignment). C - NrqD-MESO_PHOSAC3v1_90503 (62 sequences, 191 amino acid positions kept after trimming of the alignment). D - NrqE-MESO_PHOSAC3v1_90504 (62 sequences, 189 amino acid positions kept after trimming of the alignment). E - NrqF-MESO_PHOSAC3v1_90505 (62 sequences, 351 amino acid positions kept after trimming of the alignment).

Fig. S5. Sequence Alignment of the RnfB subunit from Rnf complexes of the Thermotogae members. The sequences were aligned with MAFFT. Conserved positions are indicated with colours. Accession number of sequences: 
Pseudothermotoga lettingae TMO (YP_001469925), Pseudothermotoga thermarum DSM 5069 (YP_004660443), Petrotoga mobilis SJ95 (YP_001568014), Marinitoga piezophila KA3 (YP_005096257), Kosmotoga olearia TBF 19.5.1 (YP_002940621), Thermotoga naphthophila RKU-10 (YP_003346384), Mesotoga prima MesG1.Ag.4.2 (Theba1348), Mesotoga prima PhosAc3 (MESO PHOSAC3v1_120293), Mesotoga infera VNs100 (MESINFAv2 2581), Thermotoga naphthophila RKU-10 (YP_003346384), Thermotoga petrophila RKU-1 (YP_001244271), Thermo- toga neapolitana DSM 4359 (YP_002533979), Thermotoga maritima MSB8 (NP_228063), Fervidobacterium pennivorans DSM 9078 (YP_005471998), Fervidobacterium nodosum Rt17-B1 (YP_001411073), Thermosipho melanesiensis Bl429 (YP_001306837) and Thermosipho africanus TCF52B (YP_002335587).

Table S1. List of the 2775 prokaryotic proteomes used in the phylogeny analyses. 